\title{
Review of: "Dynamic-boundary-based lateral motion synergistic control of distributed drive autonomous vehicle"
}

Rodolfo Haber

Potential competing interests: The author(s) declared that no potential competing interests exist.

This paper presents a cooperative lateral motion control method based on the dynamic boundary in order to consider different road adhesion conditions from affecting the motion stability. The theoretical development is supported on well known equations and the claimed novelty relies on a dynamic boundary considering the pavement adhesion coefficient for considering stable and unstable regions. A layered control architecture is proposed. Whilst the theoretical foundations for lateral control presented in the work are promising the simulation study, carried out on MatLab/Simulink, does not sufficiently demonstrate the actual impact of the proposed control solution before real driving scenarios as reported so far for example some years ago by J. Godoy, et al. "A driverless vehicle demonstration on motorways and in urban environments," Transport, vol. 30, no. 3, 2015, doi: 10.3846/16484142.2014.1003406, and more recently by Artunedo, A., Villagra, J., Godoy, J. in IEEE Transactions on Intelligent Transportation Systems in the seminal paper "Jerk-Limited Time-Optimal Speed Planning for Arbitrary Paths". 